\title{
Muzlink: Three Level Connected Timelines for Artist-Centered Musical Adaptations Exploration
}

\author{
François Lévesque* \\ Polytechnique Montreal
}

\author{
Marielle St-Germain ${ }^{\dagger}$ \\ Bibliothèques et Archives nationales du Québec (BAnQ)
}

\author{
Thomas Hurtut \\ Polytechnique Montreal
}

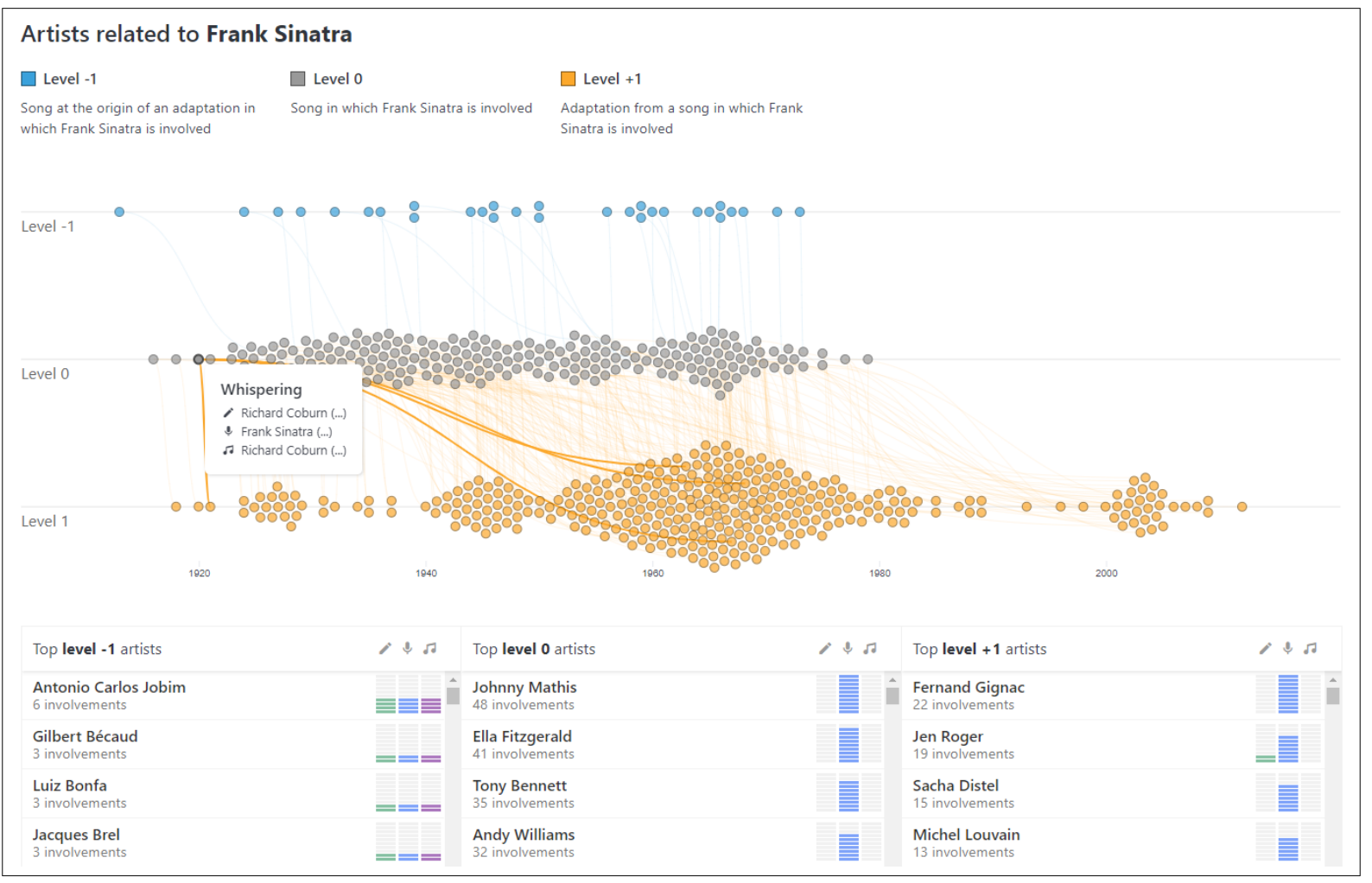

Figure 1: The Muzlink explorer allow laypersons to discover songs and artists who collaborated, starting from a query artist. Here, we browse Frank Sinatra's body of songs over time as grey circles (level 0 timeline). Blue circles (level -1 timeline) are the songs at the origin of a musical adaptation in which Frank Sinatra is involved. Conversely, orange circles (level +1 timeline) are the songs adapted from Frank Sinatra's. Muzlink thus let explore how and when an artist got inspired, collaborated or inspired other artists. The tooltip on the song node "Whispering" shows that it has been written and composed by Richard Coburn, and sung by Sinatra. Edges towards four direct adaptations of the song are highlighted. On the bottom linked view, top artists related to Sinatra are listed and can be filtered by role (songwriter, composer, singer).

\section{ABstract}

This design study tackles the visualization of a multivariate bipartite graph of artists (songwriters, composer, singer) and songs. Furthermore, this dataset has an additional tree structure for the song nodes, inherited from the musical adaptation relations. In tight collaboration with a national library and archives agency, we propose an artist-centered interactive representation, focusing on several identified user tasks. Data and task abstraction, design considerations, and a preliminary insight-based case study are presented.

Index Terms: Information visualization, design study, musical adaptations, bipartite graph, multivariate graph.

*e-mail: francois.levesque@polymtl.ca

†e-mail: marielle.st-germain@umontreal.ca

†e-mail: thomas.hurtut@polymtl.ca

\section{BACKGROUND AND DATA/TASK ABSTRACTION}

This design study presents a research work conducted in collaboration with Bibliothèque et Archives nationales du Québec (BAnQ), which is the national library and archives agency of the Quebec government. This institution has a dataset of around 24000 songs. For each song, metadata focuses on the artists who were involved in the production of each song.

In this dataset, a song gathers artists bearing three different possible roles (songwriter, composer, singer). Each role can be hold by multiple artists (e.g. if two songwriters coauthored a song). This dataset can thus be structured first into a bipartite graph, with artist nodes on a side, and song nodes on the other. Each edge in this graph has an artist role, sometimes being multidimensional. For instance, Richard Coburn both wrote and composed the song Whispering, sung by Frank Sinatra (see Figure 1). The BAnQ dataset gathers around 19000 artists. Besides, a song's lyrics can be adapted, either in another language, or simply into another version. This aspect lets us structure the song nodes into tree structures based on these adaptation links. The BAnQ dataset has a strong focus on this aspect 
since all songs have either a parent or a child node. The average number of songs per tree is 3.4 in the dataset, and the maximum depth of an adaptation tree is 4 . Following Munzner data abstraction [3], the BAnQ dataset is thus an unusual combination of a bipartite multivariate graph and an overlayed tree structure.

The design process conducted in collaboration with BAnQ, led to the proposition of an interactive visualization of this dataset, described in Section 3. The proposed design targets several tasks, identified at the beginning of this process. Overall, the intended users are the BAnQ website visitors, willing to discover and enjoy the sometimes unexpected relations that may exist between artists and songs. BAnQ sees that exploration, or high-level consume task [3], as a possible stepping stone for a library borrowing of a physical compact disc. Specifically, starting from an artist query they know, a user should be able to browse the distribution of his songs over time, and the songs from/to which they have been adapted. He should also be able to perform different kind of search actions on these bodies of songs, such as locate the artists with who their query collaborated the most and identify their roles, lookup, and identify artists' outliers.

\section{Previous work}

As the dataset combines a bipartite graph and a tree structure, previous works focusing on graph such as PivotPaths [1], are usually partially fitted. The tasks primarily revolve around the tree structure of the songs. Therefore, we initially reviewed existing techniques of tree representations: node link, nested boxes, indented list and matrix [2]. A node link representation explicitly reflects the topology but is not ideal for large trees with many heterogeneous node attributes [5]. However, an attribute-driven grouping and positioning can overcome this last limitation on sparse networks [6]. A nested box allows more nodes to be displayed but the topology is harder to perceive. An indented list view makes the structure relatively simple to understand but needs larger screen space to do so. A matrix representation is well suited for large trees and complex node attributes, but it is not ideal for visualizing tree structures [5]. An integrated representation combining a topological and an attribute view is relevant for our targeted tasks. Juniper is an interesting recent approach combining an indented list view and a table view for easier multivariate graph analysis [4]. This tool is especially powerful to visualize complex relations between neighbor's nodes. However, it lacks a way to show both the tree and the bipartite graph structures at once.

\section{MUZLINK}

\section{Design Considerations}

Since artists relationships are supported by the songs and their musical adaptations, they constitute the base unit of the proposed visualization. Furthermore, the dataset's large size and structure constrain to represent one artist at a time. After filtering by a given artist, we are left with a number of small adaptation trees for which at least one node is related to the artist. For each song in the trees, it's then possible to find all related artists.

Since an artist can bear many roles for each song, adaptation relations are complex. Well defined categories were created to better communicate them: level -1 , level 0 and level +1 . A level -1 song is at the origin of adaptation in which the selected artist is involved. A level 0 song is a song in which the artist is involved. A level +1 song is an adaptation of a song in which the artist is involved.

All trees are visualized as node links. Nodes are vertically positioned and colored according to their level and horizontally positioned by their release year. The node link was chosen for its expressive topological representation. The attribute-driven grouping allows to quickly reflect the relationship nature between songs [5]. The timeline positioning intuitively encodes when those relationships took place over time.
Then, adding edges effectively creates connected timelines. All edges are drawn with a low opacity and a small bezier curve in order to reduce the visual clutter when the graph is dense. With such a layout, it becomes difficult to distinguish individual trees, but the accumulation of edges provides rich visual cues regarding the trend and distribution of the relationships.

At the bottom, a table lists the most involved artists for each level. In a small multiple fashion, small bar charts are laid out next to each artist. These charts present the role distribution of the artist for a single level. Standard bar charts were chosen for the high precision at which they can visually express data [3].

\section{Interaction}

When a song is hovered, its parent and children, if any, are highlighted along with the involved edges. A small tooltip also reveals the title of the song, as well as the various artists involved. When an artist from the bottom lists is hovered, all nodes and related edges in which the artist is involved are highlighted. For each role (author, singer \& composer), a clickable icon on the list header allows to sort the artists by role. Every artist visible in the view is clickable, leading to its Muzlink centered view, in order to improve the exploration task.

\section{Implementation}

A complete implementation for desktop is available at http:// muzlink.witify.io. The tool was developed with the $d 3 . j s$ and vue.js libraries, entirely in javascript.

\section{Preliminary Case Study}

BAnQ recently used Muzlink during a Galleries, Librairies, Archives and Museums (GLAM) event in Montreal gathering professionals from the cultural industry. 7 persons were confronted to the proposed visualization during an open demo session. These users were allowed to ask questions guiding the exploration of the data. Overall, the feedback was very positive from all these participants, stressing the innovative way to represent such linked data, and its exploration potential. For instance starting from a famous Quebec rock band from the sixties called Les Baronets, a user discovered that they adapted a lot of the Beatles' over their carreer.

\section{Conclusion ANd Future Work}

Because of the complexity of their relations, artists involved in song production and musical adaptations offer a challenging information visualization problem. We propose an artist-centered design based on connected timelines to tackle this challenge. Although we presented a case study on musical data in the context of a collaboration with a public library and archives agency, this research work could be useful for the exploration of other datasets, such as movie datasets, citation networks, versions of historical documents etc. Future work will include first a thorough user study with a panel of BAnQ onsite library visitors. Besides, adding another layer of complexity by including the musical arrangement (modification of the song composed work) would be an interesting new research perspective.

\section{REFERENCES}

[1] M. Dörk, N. H. Riche, G. Ramos, and S. Dumais. Pivotpaths: Strolling through faceted information spaces. IEEE TVCG, 18(12), 2012.

[2] M. Graham and J. Kennedy. A survey of multiple tree visualisation. Information Visualization, 9(4), 2010.

[3] T. Munzner. Visualization analysis and design. CRC Press, 2014.

[4] C. Nobre, M. Streit, and A. Lex. Juniper: A tree+table approach to multivariate graph visualization. IEEE TVCG, 25(1), 2019.

[5] C. Nobre, M. Streit, M. Meyer, and A. Lex. The state of the art in visualizing multivariate networks. CGF (EuroVis '19), 38, 2019.

[6] B. Shneiderman and A. Aris. Network visualization by semantic substrates. IEEE TVCG, 12(5), 2006. 